\title{
Identificando Demandas Tecnológicas Computacionais para Atender as Necessidades do Cidadão: Um Estudo de Caso na Cidade de Niterói
}

\author{
Raissa Barcellos ${ }^{1}$, Flavia Bernardini ${ }^{1}$, José Viterbo ${ }^{1}$, Luiz Monteiro ${ }^{2}$, Marília Ortiz ${ }^{2}$ \\ ${ }^{1}$ Instituto de Computação - \\ Universidade Federal Fluminense (UFF) - \\ Niterói - RJ - Brasil \\ ${ }^{2}$ Prefeitura Municipal de Niterói \\ \{rbarcellos, fcbernardini, viterbo\}eic.uff.br \\ \{luizj,marilia\}@seplag.niteroi.rj.gov.br
}

\begin{abstract}
Transparency in local governments should take into account popular participation to identify data and services demands. Assessing the demand of the population can be done through living labs. The objective of this work is to present a case study in which (i) we analyzed the demands presented at the 2 nd Conference on Transparency and Social Control in the city of Niteroi (execution of activities in a living lab) and (ii) we presented the need for infrastructure structure of ICTs and necessary computer systems. We observed that it is still necessary to carry out a study to identify which ICT projects must be executed by the local government to achieve the pointed demands.
\end{abstract}

Resumo. A transparência nos governos municipais deve levar em coinsideração a participação popular para identificar demandas de disponibilização e oferta de dados e serviços. Aferir a demanda da população pode ser realizada por meio de laboratórios vivos. O objetivo deste trabalho é apresentar um estudo de caso no qual (i) analisamos as demandas apresentadas na 2 a Conferência de Transparência e Controle Social na cidade de Niterói (execução de atividades em um laboratório vivo) e (ii) apresentamos a necessidade de infra-estrutura de TICs e sistemas computacionais necessários. Ainda é necessário um estudo para identificar quais projetos de TIC precisam ser executados para atender as demandas apontadas.

\section{Introdução}

Transparência é a extensão em que um órgão público é visível ao público no que diz respeito a seus processos. Refere-se a requisitos relativos à qualidade da manutenção de registros, disseminação ativa e generalizada, com facilidade de acesso a documentos relevantes, registros, pontualidade e regularidade da resposta às solicitações, abrangência, usabilidade e qualidade dos registros mantidos ou fornecidos [Sridhar et al. 2020]. Para que a transparência seja alcançada, entendemos que a participação do público deva ser permitida. Segundo [Sridhar et al. 2020], os estudos de governança com foco na participação do público são abundantes, embora sua inclusão pelos governos continue sendo limitada. Persons [Persons 1990] enfatizou que a legitimidade da formulação de 
políticas no nível regional, assim como nos níveis mais convencionais do governo, dependia da qualidade da representação, ressaltando que os cidadãos e comitês voluntários tendem a perder valor quando estão confinados aos costumes habituais. O argumento a favor da participação do público decorre do fato de que os leigos também podem tomar decisões informadas sobre questões técnicas que abrangem o nível regional, desde que as informações relevantes sejam compartilhadas da maneira apropriada, devido às quais os governos devem procurar legitimar sua participação.

As modernas tecnologias da informação e comunicação constituem uma base essencial e são consideradas os principais impulsionadores do governo aberto. Com a participação popular, as demandas são mais claras, a fim de implantar tecnologias mais específicas. Por exemplo, portais governamentais ou mídias sociais públicas podem ser usadas não apenas para publicação oportuna de dados abertos do governo, mas também para participação do cidadão e interação em tempo real entre instituições públicas e cidadãos. É particularmente esse desenvolvimento tecnológico em conjunto com o governo aberto que traz uma variedade de benefícios para o governo e a sociedade [Wirtz et al. 2018]. Além disso, por meio de atividades tipicamente realizadas em laboratórios vivos, como o levantamento de demandas em conferências abertas à participação popular, são particularmente interessantes para assessar a opinião da população. Tais demandas apontadas muitas vezes requerem a execução de projetos estruturantes para atender a requisitos de Tecnologias da Informação e Comunicação e Sistemas Computacionais, ou ainda realizar uma análise do status atual das TICs existentes no governo, para verificar se podem ser adaptadas para atenderem tais demandas.

O principal objetivo deste trabalho é, a partir de um estudo de caso realizado na $2^{\text {a }}$ Conferência de Transparência e Controle Social de Niterói, propor alternativas de ferramentas computacionais aptas a amparar o Governo de Niterói na implantação de demandas populacionais. A agenda da $2^{a}$ Conferência de Transparência e Controle Social de Niterói foi construída por meio de coleta de informações na realização de uma conferência organizada pela prefeitura e a sociedade civil. Coletamos dados em duas etapas da conferência. A partir dessa coleta, apresentamos as tecnologias necessárias de TICs e sistemas computacionais capazes de apoiar as demandas apontadas pelos cidadãos. Como próximos passos desse trabalho, é necessário identificar quais das tecnologias apontadas podem ser suportadas pela infra-estrutura de TIC atual na prefeitura.

Este trabalho está organizado da seguinte forma: Na Seção 2 é apresentado um referencial teórico sobre transparência pública e transparência de dados, dados abertos e laboratórios vivos. Na Seção 3 é apresentada uma revisão da literatura sobre como assessar a população para obter opinião das demandas para transparência. Na Seção 4 é apresentada a metodologia para condução do nosso estudo de caso, realizado na com base nas atividades da $2^{\text {a }}$ Confereência de Transparência e Controle Social. Na Seção 5 são apresentadas as demandas de TIC e sistemas computacionais necessárias para atender às necessidades do cidadão apontadas na conferência. Finalmente, na Seção 6 são apresentadas nossas conclusões e trabalhos futuros. 


\section{Referencial Teórico}

\subsection{Transparência Pública e Dados Governamentais Abertos}

A OECD (Organisation for Economic Co-operation and Development) considera a transparência um princípio fundamental na construção de um governo aberto, significando "informações confiáveis, relevantes e oportunas sobre as atividades do governo disponíveis ao público". Exemplos de transparência não são novos. Em 1953, um relatório abrangente já havia sido publicado sobre leis e decisões judiciais que tratavam do direito de acesso à informação [Corrêa et al. 2017].

Logo, a partir de definições distintas e não formais na literatura, [Cappelli 2009] buscaram referências com o intuito de propor uma definição formal que possibilitasse estruturar, de forma eficiente, o conceito de transparência. Com isso, foram criados cinco principais grupos de características e se percebeu uma dependência entre eles. Os grupos são: acessibilidade, usabilidade, informativo, entendimento e auditabilidade. [Cappelli 2009] concluem que, para uma boa capacidade de transparência, a informação deve ser acessível, usável, informativa, entendível e auditável. Com a apresentação de todas estas características, é definido um conceito completo de transparência em um contexto geral, e mais especificamente no contexto social.

Segundo Jetzek et al. [Jetzek et al. 2019], o termo dados abertos refere-se principalmente aos dados que foram criados ou coletados pelas agências governamentais para uma finalidade, e que são disponibilizados ao público para outras finalidades. As agências governamentais geram e retém uma extensa gama de dados valiosos, que comumente estariam fora do alcance de terceiros. Com a abertura desses dados, diversas comunidades diferentes podem usá-los simultaneamente para diversos fins. Governos e organizações internacionais manifestaram perspectivas de que a abertura do acesso aos seus dados facilitaria a criação de relevantes benefícios para a sociedade, organizações e indivíduos [Jetzek et al. 2019].

O movimento de dados abertos é o primeiro passo para levar à transparência pública [Bernardini et al. 2017]. A rogativa para uma maior abertura e transparência tem sido percebida pelos governos de todo mundo. Atualmente, há diversas reuniões governamentais, debates, campanhas e processos judiciais para trazer uma maior responsabilidade aos governos diante dos cidadãos. No contexto brasileiro, em 2011 foi sancionada a lei de acesso à informação —- lei $\mathrm{n}^{0}$ 12.527, de 18 de novembro de 2011 —-, que define que, dentre outros tantos aspectos, as informações devem ser disponibilizadas de maneira primária, íntegra, autêntica e atualizada em todos os órgãos públicos, empresas de economia mista, dentre outros, na União, Estados, Distrito Federal e Municípios. No entanto, ainda há um longo caminho para que os dados considerados públicos estejam disponibilizados para a população.

De acordo com [Alzamil and Vasarhelyi 2019], alcançar a transparência em dados governamentais abertos pode ser muito desafiador. A transparência pode desempenhar um papel primordial na tomada de decisões dos governos. Ao disponibilizar os dados do governo ao público, cidadãos, profissionais e outros grupos interessados podem acessar os dados para ajudar a monitorar os gastos públicos e aumentar a participação geral, permitindo assim que funcionários do governo tomem melhores decisões. Além de uma melhor 
tomada de decisão, a transparência pode ajudar a evitar ou minimizar o abuso de recursos do governo. Quando funcionários do governo sabem que seu trabalho está sendo monitorado, é menos provável que cometam erros [Alzamil and Vasarhelyi 2019]. No entanto, para que a disponibilização de dados e informações ocorra, é necessário entender a demanda da população relativa a quais dados e informações precisam ser disponibilizados e, ainda, identificar quais TICs e sistemas computacionais são necessários.

\subsection{Laboratório Vivo}

De acordo com [Ruijer and Meijer 2019], um laboratório vivo pode ser definido como ambientes para inovação orientada ao usuário. Os laboratórios vivos oferecem uma plataforma colaborativa para pesquisa, desenvolvimento e experimentação em ambientes da vida real, com base em metodologias e ferramentas específicas, e implementadas através de projetos específicos de inovação e atividades de construção da comunidade. Eles são descritos pela experimentação em configurações do mundo real e pelos usuários como co-inovadores. Os usuários colaboram para criar o resultado desejado. Os laboratórios vivos oferecem uma alternativa aos experimentos da administração pública que geralmente são realizados em um ambiente controlado de laboratório [Bouwman and Grimmelikhuijsen 2016].

Novas configurações sociais são necessárias para a organização da inovação onde o governo, indústria, academia e cidadãos trabalhem juntos para co-criar o futuro e conduzir mudanças estruturais, muito além do escopo do que qualquer organização ou pessoa poderia fazer individualmente [Carayannis and Campbell 2009]. A utilização de um laboratório vivo se mostra como uma excelente oportunidade para os órgãos públicos se encontrarem com pessoas e outras organizações, a fim de experimentar juntos novos conceitos e possibilidades [Manzini and Staszowski 2013].

\section{Revisão da Literatura}

Alguns trabalhos presentes na literatura já realizaram estudos a fim de compreender e avaliar mecanismos de acesso à opinião da população, e estimular o empoderamento do cidadão ao co-participar de decisões governamentais. Em [Bernardini 2017], a autora apresenta um estudo de caso que avalia o mecanismo de acesso às informações da população, para definição dos rumos do projeto de cidades inteligentes, para o município de Rio das Ostras. É apresentado um método para coleta das opiniões, bem como uma análise das opiniões e ideias sugeridas.

Já em [Capra 2016], o trabalho mostra a presença de uma relação entre estruturas de governança e tipologias de participação ativa do cidadão em Amsterdam. A pesquisa examina as características de governança presentes no programa de cidade inteligente e em uma amostra selecionada de projetos, e como elas se relacionam com diferentes tipologias de participação cidadã. $\mathrm{O}$ autor alega que a participação na tomada de decisões envolve não apenas cidadãos e comunidades, mas também atividades econômicas locais. O estudo permitiu uma melhor compreensão da dinâmica da governança dos programas de cidade inteligente de Amsterdam.

Em [Thiel et al. 2017], o autor apresenta informações sobre a construção e a avaliação de um aplicativo móvel gamificado, implantado em uma cidade da Finlândia. O aplicativo móvel, que serviu como banco de ensaio para o o estudo, incluiu vários elementos diferentes de gamificação, que foram adicionados com o objetivo de incentivar 
os cidadãos a usar ferramentas digitais que visam facilitar a comunicação entre eles e as autoridades da cidade. Nesse trabalho, o autor também relata a necessidade de promover o interesse do cidadão no planejamento urbano, através da construção da confiança no impacto da participação pública. Quando existe essa motivação prévia, as ferramentas utilizadas atendem ao objetivo pretendido: facilitar a participação do público, em vez de criá-la.

\section{Estudo de caso}

A fim de realizar nosso estudo de caso, participamos da $2^{a}$ Conferência de Transparência e Controle Social ( $2^{\mathrm{a}}$ CONSOCIAL) ${ }^{1}$, que teve como propósito construir junto à sociedade civil de Niterói uma agenda de propostas para aprimorar a Política Municipal de Transparência, criada pela Lei Municipal no 3188, de 21 de dezembro de 2015. Esta lei criou também o Conselho Municipal de Transparência e Controle Social, que tem atuado desde 2016 como ator relevante na proposição de pautas e avanços na gestão municipal da transparência e foi também uma das instituições organizadoras da $2^{\mathrm{a}}$ CONSOCIAL.

A $2^{\text {a }}$ CONSOCIAL ocorreu em duas etapas: uma pré-conferência, dia 14 de setembro de 2019; e uma conferência de encerramento, no dia 12 de outubro de 2019 . Na pré-conferência, o foco foi direcionado aos Conselhos de Políticas Públicas, onde os cidadãos foram motivados a realizar uma reflexão sobre o papel de instituições públicas, contribuindo com opiniões e elementos concretos, a fim de influenciar a formulação e exercer o controle social das políticas municipais. Já a conferência de encerramento funcionou como um laboratório vivo presencial para que, juntamente aos cidadãos, a prefeitura de Niterói conduza mudanças nas áreas de Transparência de dados e Controle social. Na conferência de encerramento foram debatidos temas como: (i) governo eletrônico, (ii) dados abertos, (iii) mecanismos e instâncias de participação social e (iv) informação como um direito e governo aberto, a fim de consolidar uma gama de propostas, aprimorando a política municipal de transparência e controle Social. A coleta de dados, em ambas as etapas, foi realizada através de um fórum de discussão e debates em grupos de trabalho, a fim de viabilizar a comunicação eficiente entre Governo e cidadão.

\subsection{Perfis dos participantes}

Na pré-conferência foram coletados dados como município de residência de cada participante - foram 212 no total - e instituição no qual cada participante reside. De acordo com a Figura 1(a), observamos que a maioria participantes reside no município de Niterói. Também, de acordo com a Figura 1(b), observamos que a maioria dos participantes faz parte de organizações voluntárias atreladas ao município. Funcionários da Prefeitura Municipal de Niterói (PMN) também faz parte de uma grande parcela dos participantes do evento.

Já nas Figuras 2(a) e 2(b), são mostradas as características dos participantes na conferência de encerramento - foram 150 no total. Podemos observar que a maior parte dos participantes da conferência de encerramento é residente do município de Niterói e funcionário da Prefeitura Municipal de Niterói (PMN).

\footnotetext{
${ }^{1}$ http://conferenciatransparencia.niteroi.rj.gov.br/conf2019/
} 


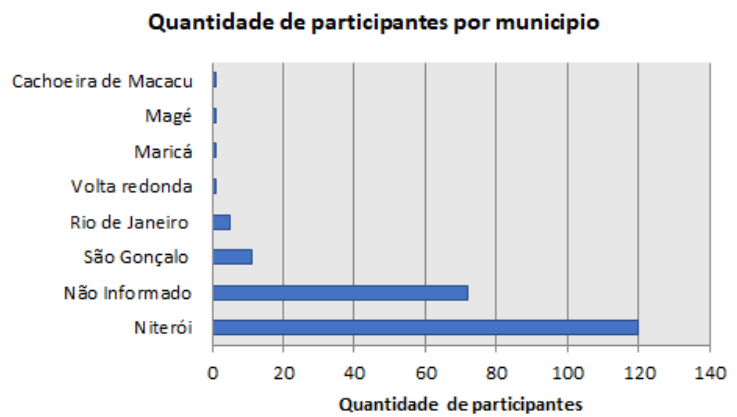

(a) Quantidade de cidadãos por município

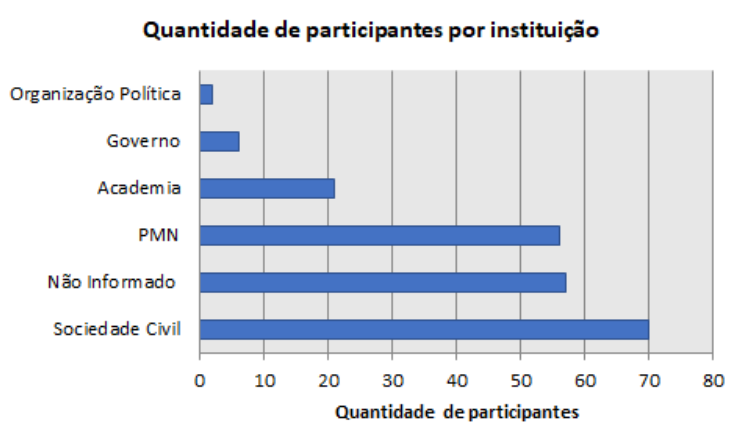

(b) Quantidade de cidadãos por instituição

Figura 1. Perfil dos Participantes na Pré-Conferência

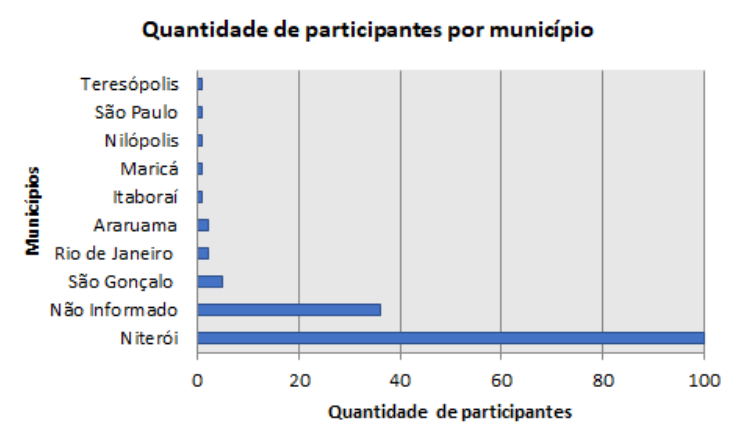

(a) Quantidade de cidadãos por município

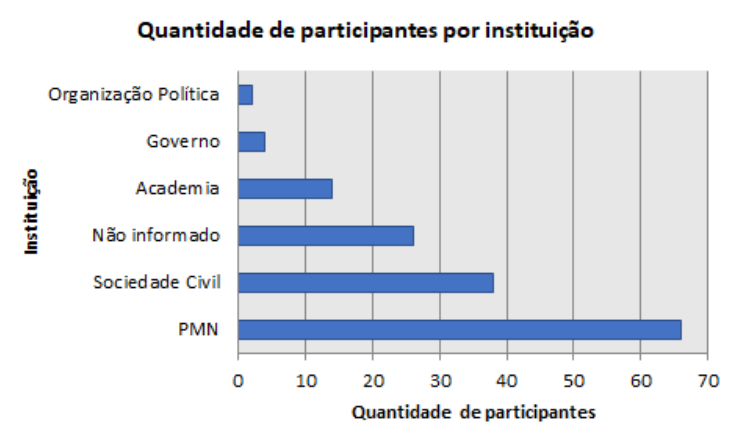

(b) Quantidade de cidadãos por instituição

Figura 2. Perfil dos Participantes na Conferência de Encerramento

\subsection{Propostas aprovadas}

A partir do laboratório vivo realizado pela conferência, uma série de propostas foram aprovadas, com o intuito de influenciar a implementação das mesmas para avançar na construção de um governo aberto em Niterói ${ }^{2}$. Nesta pesquisa, separamos as propostas relativas à Ciência e Tecnologia em seis grupos.

1. Visualizações de dados

(a) Facilitar o acesso às informações dos investimentos públicos, com gráficos e comparativos (séries históricas)

2. Usabilidade/Acessibilidade de portais

(a) Melhorar acessibilidade de conteúdos nos sítios eletrônicos para pessoas com deficiência;

(b) Promover testes de navegação nos portais com diferentes públicos, analisando a usabilidade de modo a implementar um sistema de avaliação desses portais;

(c) Sistema de informação para o público com acessibilidade. Acesso através de totem celular, centro de atendimentos e universidades

3. Comunicação Digital

\footnotetext{
${ }^{2}$ Todas as propostas aprovadas podem ser encontradas em http:// conferenciatransparencia.niteroi.rj.gov.br/conf2019/wp-content/uploads/ sites/3/2019/10/Propostas_Aprovadas_Conferencia.pdf.
} 
(a) Ampliar o uso das redes sociais, e-mail e whatsapp para divulgar o portal da transparência e suas atualizações

4. Informações em tempo real

(a) Criar novo portal de transparência que contenha consultas mais dinâmicas, dados abertos e informações atualizadas;

(b) Transparência em tempo real dos serviços públicos aos usuários dos serviços, como p. ex. "qual a posição do cidadão na fila da saúde", "prématrículas das unidades de educação da rede municipal"”, dentre outros;

(c) Criar plataforma, que disponibilize dados, em tempo real, acerca dos serviços básicos, como p. ex. vagas escolares, consultas, exames, cirurgias, dentre outros;

(d) Garantir que os serviços prestados por agentes privados sejam controlados em tempo real pela administração e por segmentos organizados, cabendo ao prestador emitir relatórios com os serviços fornecidos em determinado período

5. Acesso à internet

(a) Garantir a conectividade a todos os equipamentos da prefeitura municipal: escolas, hospitais, bibliotecas e ampliação da inclusão digital das comunidades;

(b) Democratizar o acesso à internet, ampliando o WiFi público e promovendo formações permanente sobre governo digital e transparência para os cidadãos, usando os telecentros

6. Aplicativos e sistemas de informação integrados

(a) Disponibilizar serviços online de saúde, como marcação de consultas, exames entre outras funções, facilitando o acesso da população;

(b) Construir uma solução de diário oficial aberto;

(c) Abrir dados relativos ao transporte público para desenvolvimento de aplicativos ou outras soluções tecnológicas que melhorem o serviço de transporte;

(d) Criar sistema único de acesso às compras públicas com transparência;

(e) Construir um Plano de Transformação Digital que implante um setor ou empresa pública municipal de tecnologias da informação e de inovação, garantindo a disponibilidade e a confiabilidade das redes de eletricidade e de comunicação para cidadãos de todas faixas de renda e áreas da cidade -em especial nos equipamentos e espaços públicos, orientando os sistemas de informação do município a se comunicarem por APIs entre si e com a sociedade e prevendo a formação de equipes e profissionais de segurança cibernética trabalhando junto à Prefeitura;

(f) Facilitar o acesso do cidadão ao Poder público, por meio de plataformas ou aplicativos intuitivos e com linguagem simples, bem como criando "Casas da Transparência Cidadã” nas comunidades vulneráveis de Niterói, fortalecendo a cultura da transparência e o controle social

\section{Demandas de Ferramentas Computacionais para Atender as Necessidades}

Para cada grupo, pesquisamos na literatura, alternativas de ferramentas computacionais que são capazes de auxiliar a Prefeitura de Niterói a implementar as propostas aprovadas na conferência. 
Visualizações de Dados: Para implementar diversos tipos de visualizações de dados a partir dos dados provenientes do portal de dados governamental aberto de Niterói, há a possibilidade de utilizar bibliotecas Python, como Seaborn ${ }^{3}$, Bokeh $^{4}$ e Altair ${ }^{5}$. Essas bibliotecas também podem ser aplicadas juntamente à solução de aplicativos e sistemas de informação que façam uso dos dados públicos disponibilizados.

Usabilidade/Acessibilidade de Portais: De acordo com Nielsen [Brooke et al. 1996], Usabilidade é "a capacidade de aprender e memorizar um sistema de software, a sua eficiência de uso, a sua capacidade de evitar e gerenciar erros de usuário e satisfação do usuário". Já acessibilidade é, por definição, uma categoria de usabilidade: o software que não é acessível a um usuário específico não é utilizável por essa pessoa [Bergman and Johnson 1995].

Usabilidade e acessibilidade estão entre os critérios de qualidade mais críticos para sites do governo para fornecer melhores serviços a todos os seus cidadãos, incluindo pessoas com deficiência e idosos. Eles também são muito cruciais para os governos disseminarem informações e serviços em todos os cidadãos, uma vez que os usuários não preferem visitar um site novamente no futuro se enfrentarem uma interface complexa e características de baixa usabilidade. Portanto, os desenvolvedores precisam planejar os portais conforme às questões de usabilidade dos sites governamentais, a fim de projetar e desenvolver produtos eficazes, eficientes e satisfatórios e tornar os serviços governamentais acessíveis a um grande número de cidadãos [Karaim and Inal 2019]. É de extrema importância a implantação, por especialistas da área de interação humano-computador, de uma metodologia de avaliação de usabilidade e acessibilidade, para garantir que esses dois critérios sejam respeitados na construção de portais governamentais.

Comunicação Digital: A mídia de comunicação digital oferece alguma conveniência prática que não pode ser alcançada por conversas verbais presenciais [Chan and Sage 2019]. Na prática, a mídia social serve como uma frase abrangente para um conglomerado de tecnologias e serviços baseados na Web, como blogs, microblogs (Twitter), serviços de compartilhamento social (por exemplo, YouTube, Flickr, StumbleUpon, Last.fm), mensagens de texto, fóruns de discussão, ferramentas de edição colaborativa (por exemplo, wikis), mundos virtuais (por exemplo, Second Life) e serviços de redes sociais (por exemplo, Facebook, MySpace).

A interação entre o governo e seus cidadãos nas mídias sociais pode ser examinado através de tecnologias de análises de redes sociais. O trabalho [Idris 2018], utiliza um método de análise de redes sociais para analisar quatro tipos de conversas entre o governo da Indonesia e seus cidadãos nas mídias sociais: (i) comunicação diária, (ii) campanha de comunicação, (iii) crise e (iv) comunicação de emergência.

Segundo [Bertot et al. 2012], o emprego das mídias sociais no governo oferece várias oportunidades, como:

- Participação e engajamento democráticos, usando tecnologias de mídia social para envolver o público no governo, promovendo o diálogo participativo e dando voz às discussões sobre desenvolvimento e implementação de políticas.

\footnotetext{
${ }^{3}$ https://seaborn.pydata.org/

${ }^{4} \mathrm{https} / / /$ docs.bokeh.org/en/latest/index.html

${ }^{5}$ https://altair-viz.github.io/
} 
- Co-produção, na qual os governos e o público desenvolvem, projetam e prestam serviços governamentais em conjunto para melhorar a qualidade, a entrega e a capacidade de resposta dos serviços.

- Soluções e inovações de crowdsourcing, buscando inovação por meio do conhecimento e talento do público para desenvolver soluções inovadoras para questões sociais de larga escala. Para facilitar o crowdsourcing, o governo compartilha dados e outras informações para que o público tenha uma base fundamental para inovar.

Logo, a utilização de mídias sociais na comunicação digital pode ser otimizada a partir do emprego de tecnologias de informação e comunicação.

Informações em Tempo Real: Na era do Big Data, processamento em tempo real recebeu uma atenção crescente, especialmente com a expansão de dados em volume e complexidade. Para garantir um processamento de informações confiável e rápido em tempo real, ferramentas poderosas são essenciais para a análise e o processamento de Big Data. A implementação do Hadoop MapReduce e da estrutura Apache Spark são tecnologias úteis para analisar fluxos de dados em tempo real [Aziz et al. 2018]. O MapReduce é um modelo de programação que permite a computação paralela em uma grande quantidade de dados usando um grande número de máquinas chamadas Cluster ou Grid (mais dados, mais máquinas). Já o Apache Spark é um mecanismo de processamento de código aberto altamente preocupado com a rapidez e a confiabilidade. Foi desenvolvido essencialmente para computação rápida. Este sistema fornece APIs em diferentes linguagens de programação, como Scala, Java e Python [Aziz et al. 2018]. Utilizar uma dessas tecnologias no backend de aplicações de portais de dados governamentais abertos, a fim de disponibilizar informações em tempo real ao cidadão, pode ser uma alternativa prática e eficiente.

Acesso à Internet: $\mathrm{O}$ desenvolvimento avançado do acesso à banda larga é um passo inevitável em direção à vida diária de suporte e ao crescimento econômico sustentável. É essencial promover o desenvolvimento de um ambiente rico de redes de banda larga que suportam aplicativos digitais, garantindo que essas redes estejam disponíveis em toda a cidade. Esse imperativo é crítico porque as cidades não precisam apenas se concentrar na construção de infraestrutura para suportar tecnologias conectadas, mas também precisam garantir que todos os residentes e empresas tenham acesso à banda larga de alta velocidade [Young 2017].

Para alcançar velocidades aprimoradas, a maioria das grandes telecomunicações existentes está atualizando suas redes instalando novos cabos de fibra ótica de alta capacidade e/ou fazendo melhorias em seus cabos coaxiais existentes por meio de um novo padrão chamado DOCSIS 3.1.63. É importante entender que o primeiro é uma maneira muito mais sustentável e eficaz de atualizar a infraestrutura de banda larga do que o último. Os cabos de fibra ótica são a tecnologia ideal para suportar redes de banda larga de alta velocidade e alta capacidade em nossas cidades por dois motivos principais. Primeiro, a fibra possui latência menor do que qualquer outro tipo de conexão de banda larga - o que permitirá novas inovações em comunicação por vídeo, realidade virtual e realidade aumentada. A segunda fibra é mais à prova de futuro do que qualquer outra tecnologia no mercado, incluindo satélites e cabos atualizados. A fibra tem capacidade praticamente ilimitada e a infraestrutura em si dura muito tempo com custos 
limitados de manutenção [Young 2017]. Disponibilizar cabos de fibra ótica de alta capacidade para garantir conectividade em áreas e instituições públicas é um primeiro passo para democratização da internet para a cidade de Niterói.

Aplicativos e Sistemas de Informação Integrados: Comercialmente já existem soluções de integração estruturada de aplicativos e sistemas de informação que surgiram com o objetivo de facilitar a integração destes. Em 1970 surgiu uma técnica chamada Electronic Data Interchange (EDI). Essa técnica permitiu que empresas de negócios separadas se integrassem usando interfaces padrão predefinidas. O EDI foi seguido pelo Enterprise Resource Planning (ERP) na década de 1980. O ERP usou um banco de dados único para integrar todos os aplicativos que pretendiam ser integrados. Outra geração de EI foi chamada Middleware. Essa geração surgiu na década de $1990 \mathrm{e}$ usou uma camada especial para integrar diferentes aplicativos e bancos de dados. Nesse período, uma linguagem chamada XML foi descoberta e usada como linguagem padrão para comunicação na Web. Esse idioma foi usado na integração de middleware. O conceito de Enterprise Application Integration (EAI) no campo da EA foi introduzido em 1995. Essa técnica utiliza os princípios de arquitetura de software e sistemas de computador para funcionários para integrar o conjunto de aplicativos corporativos. Por meio do EAI, os desenvolvedores conseguiram integrar sistemas legados e diferentes aplicativos da web [Ramadhani et al. 2016].

A maioria dessas soluções se concentram na integração de aplicativos de diferentes setores de uma organização com o objetivo de eliminar ineficiências de comunicação [Ramadhani et al. 2016]. Com o desenvolvimento da computação em nuvem, um ERP baseado em nuvem começa a surgir como uma alternativa à solução local. Os dados e o aplicativo não são mais armazenados localmente; em vez disso, um fornecedor disponibiliza acesso ao aplicativo que pode ser personalizado para atender às necessidades do usuário e também hospeda os dados com segurança em algum lugar da Internet [Mezghani 2019]. Logo, a utilização de um ERP em nuvem para integração de aplicativos e portais de dados, seria uma solução eficaz e eficiente.

\section{Conclusões}

O objetivo deste trabalho é apresentar um estudo de caso na cidade de Niterói para levantar quais são as necessidades de infraestrutura de Tecnologias da Informação e Comunicação (TICs) e Sistemas Computacionais para atender às demandas da população. Para isso, foram coletadas informações fornecidas pelos participantes na $2^{\text {a }}$ CONSOCIAL, que equivaleu à realização de atividades de um laboratório vivo para que a prefeitura de Niterói conduza mudanças nas áreas de transparência de dados e controle social.

A partir das propostas apresentadas, realizamos um levantamento de ferramentas computacionais que podem ser utilizadas para atender as necessidades dos cidadãos. Tendo em vista que as TICs (Tecnologias da Informação e Comunicação) estabelecem um suporte para impulsionar a inicitiva do governo aberto, as tecnologias de (i) visualizações de dados; (ii) usabilidade/acessibilidade de portais, (iii) comunicação digital, (iv) informação em tempo real e (v) acesso à internet, foram as alternativas encontradas para beneficiar o governo e a sociedade, diante das propostas aprovadas no evento. Observamos que eventos como a $2^{\mathrm{a}}$ CONSOCIAL são necessários para a organização da inovação onde o governo, a academia e cidadãos trabalhem juntos, a fim de condu- 
zir mudanças e alcançar um futuro onde a Tecnologia seja intrinseca às necessidades da sociedade.

Nesse sentido, algumas ações de co-criação, como maratonas tecnológicas (hackatons), têm se mostrado importantes ferramentas na consecução do objetivo. Como exemplo, pode-se citar o HackNit, o hackaton realizado anualmente pela Prefeitura desde 2018, que tem conseguido mobilizar a sociedade civil e a academia na proposição de soluções para desafios do município. Além disso, Niterói também tem pensado na criação de fóruns permanentes de discussão, envolvendo diversos atores na construção e implementação de inovações na área de transparência do município.

Como trabalho futuro, é necessário realizar um levantamento de quais TICs estão implementadas no município de Niterói que podem auxiliar no atendimento das demandas apresentadas. Além disse, a análise de uso de boas práticas de Governança de TIC também podem ser interessantes nesse sentido.

\section{Referências}

Alzamil, Z. S. and Vasarhelyi, M. A. (2019). A new model for effective and efficient open government data. International Journal of Disclosure and Governance, pages 1-14.

Aziz, K., Zaidouni, D., and Bellafkih, M. (2018). Real-time data analysis using spark and hadoop. In 2018 4th International Conference on Optimization and Applications (ICOA), pages 1-6. IEEE.

Bergman, E. and Johnson, E. (1995). Towards accessible human-computer interaction. Advances in human-computer interaction, 5(1):87-114.

Bernardini, F. (2017). Um estudo de caso de acesso à opinião do cidadão no processo de construção e implementação de um projeto de cidades inteligentes em Rio das Ostras. In Anais do V Workshop de Transparência em Sistemas. SBC.

Bernardini, F. C., Viterbo, J., Vianna, D. S., Martins, C. B., de Medeiros, A. P., Meza, E. B. M., Moratori, P. B., and Bastos, C. A. M. (2017). General features of smart city approaches from information systems perspective and its challenges. Chapter 3, Grand Research Challenges in Information Systems in Brazil - 2016-2026. Available at https://sol.sbc.org.br/livros/index.php/sbc/catalog/ download/28/102/252-1?inline=1.

Bertot, J. C., Jaeger, P. T., and Hansen, D. (2012). The impact of polices on government social media usage: Issues, challenges, and recommendations. Government information quarterly, 29(1):30-40.

Bouwman, R. and Grimmelikhuijsen, S. (2016). Experimental public administration from 1992 to 2014. International Journal of Public Sector Management.

Brooke, J. et al. (1996). Sus-a quick and dirty usability scale. Usability evaluation in industry, 189(194):4-7.

Cappelli, C. (2009). Uma abordagem para transparência em processos organizacionais utilizando aspectos. Tese de Doutorado. PUC - Rio de Janeiro.

Capra, C. F. (2016). The smart city and its citizens: Governance and citizen participation in amsterdam smart city. International Journal of E-Planning Research (IJEPR), 5(1):20-38. 
Carayannis, E. G. and Campbell, D. F. (2009). 'mode 3'and'quadruple helix': toward a 21 st century fractal innovation ecosystem. International journal of technology management, 46(3-4):201-234.

Chan, C. and Sage, M. (2019). A narrative review of digital storytelling for social work practice. Journal of Social Work Practice, pages 1-15.

Corrêa, A. S., de Paula, E. C., Correa, P. L. P., and da Silva, F. S. C. (2017). Transparency and open government data. Transforming Government: People, Process and Policy.

Idris, I. K. (2018). Government social media in indonesia: Just another information dissemination tool. Jurnal Komunikasi: Malaysian Journal of Communication, 34(4).

Jetzek, T., Avital, M., and Bjorn-Andersen, N. (2019). The sustainable value of open government data. Journal of the Association for Information Systems, 20(6):6.

Karaim, N. A. and Inal, Y. (2019). Usability and accessibility evaluation of libyan government websites. Universal Access in the Information Society, 18(1):207-216.

Manzini, E. and Staszowski, E. (2013). Public and collaborative: Exploring the intersection of design, social innovation and public policy. DESIS network.

Mezghani, K. (2019). From on-premise erp to cloud erp. In Advanced Methodologies and Technologies in Business Operations and Management, pages 816-826. IGI Global.

Persons, G. A. (1990). Defining the public interest: Citizen participation in metropolitan and state policy making. National Civic Review, 79(2):118-131.

Ramadhani, A., Sam, A., and Kalegele, K. (2016). An overview of existing frameworks for integrating fragmented information systems. International Journal of Information, $6(3 / 4): 5$.

Ruijer, E. and Meijer, A. (2019). Open government data as an innovation process: Lessons from a living lab experiment. Public Performance \& Management Review, pages 1-23.

Sridhar, K. S., Gadgil, R., and Dhingra, C. (2020). Paving the Way for Better Governance in Urban Transport: The Transport Governance Initiative. Springer.

Thiel, S.-K., Ertiö, T. P., and Baldauf, M. (2017). Why so serious? the role of gamification on motivation and engagement in e-participation. $I x D \& A, 35: 158-181$.

Wirtz, B. W., Weyerer, J. C., and Rösch, M. (2018). Citizen and open government: an empirical analysis of antecedents of open government data. International Journal of Public Administration, 41(4):308-320.

Young, P. (2017). Broadband infrastructure to enable smart cities: Emerging strategies and partnership models. Technical Report, Georgetown University, Available at https: / / repository.library.georgetown.edu/handle/ $10822 / 1044668$. 